\title{
Integrating Wireless EEGs into Medical Sensor Networks
}

\author{
Behcet Sarikaya, M. Abdul Alim, and Siamak Rezaei \\ Department of Computer Science \\ University of Northern British Columbia \\ 3333 University Way, Prince George, BC, Canada V2M 4Z9 \\ sarikaya@ieee.org, alim@unbc.ca,siamak@unbc.ca
}

\begin{abstract}
Wireless Electroencephalograms (EEG) are currently being used to wirelessly transmit the data from brain sensors to a computer and they carry huge potential for many future medical applications. This paper presents the design of a hybrid medical sensor network with Tmote Sky motes as wireless EEG sensor nodes at the lowest level collecting EEG signals and sending them to Stargate PDAs at the next level. Stargates perform artifact removal, Fourier transformation and feature extraction and the final machine intelligence algorithms are run at a PC server. Several features of the CodeBlue medical sensor network like query processing, routing layer are used in our design. The advantages of the hybrid medical sensor network integrating wireless EEGs include the capability to have the brain monitoring functionality incorporated into the medical sensor networks.
\end{abstract}

\section{ACM Classification}

C.2.1 [Computer-Communication Networks]: Network Architecture and Design - Wireless Communication

\section{General Terms \\ Design}

Keywords

Wireless Sensor Network, Medical Sensor Network, Wireless EEG, Multihop Routing, Query Processor, Machine Learning, Tmote, Stargate

\section{INTRODUCTION}

Monitoring various signals from human body is presently an active area of research and development. Increasingly, monitoring devices are becoming wireless to allow patient mobility. Another trend is to connect monitoring devices into a network using wireless sensor nodes [1].

Wireless sensor nodes called motes consist of different types of sensors such as pulse oximeter, gyroscopes, accelerometers, an 8-bit processor and a wireless component. The processor runs a simple operating system called TinyOS

Permission to make digital or hard copies of all or part of this work for personal or classroom use is granted without fee provided that copies are not made or distributed for profit or commercial advantage and that copies bear this notice and the full citation on the first page. To copy otherwise, to republish, to post on servers or to redistribute to lists, requires prior specific permission and/or a fee.

IWCMC'06, July 3-6, 2006, Vancouver, British Columbia, Canada. Copyright 2006 ACM 1-59593-306-9/06/0007 ...\$5.00.
[2] and wireless component is based on IEEE 802.15.4 radio interface [3] for low-rate wireless personal area networks (LR-WPANs).

Real-time classification of cognitive states, such as mental tasks, may be performed using physiological measurements, such as Electroencephalograms (EEG) recordings, as predictors or correlates of those states [4]. There can be up to 24 sensors connected each constituting an EEG channel. EEGs are used in many applications such as in Brain Computer Interface (BCI) and in epilepsy treatment.

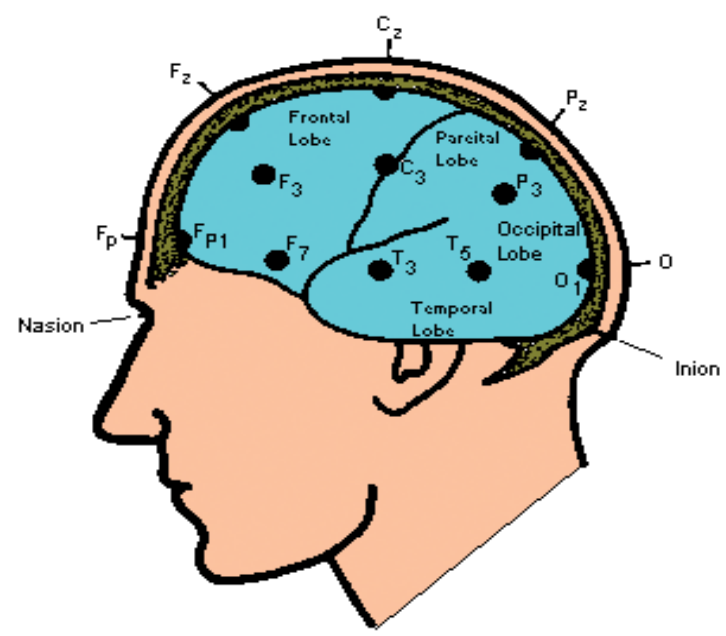

Figure 1: International 10-20 System of EEG Electrode Placement.

In this paper we discuss how EEGs can be integrated with the medical sensor networks. The next section describes the wireless EEGs. Section 3 is on the medical sensor networks, Section 4 discusses machine intelligence applications and Section 5 concludes the paper.

\section{WIRELESS EEGS}

In this section we will present the state-of-the-art in wireless EEG and then discuss the next generation EEGs. Various wireless EEGs are being offered by the biomedical industry and the product quality is improving. Wireless EEGs consist of EEG sensors, electrodes, digitizing equipment with wireless interface providing connection to a personal computer or a PDA. EEG sensors are connected to well-defined parts of the human scalp (see Fig. 1) to collect brain signals.

Brain signals from each sensor (channel) are digitized at 


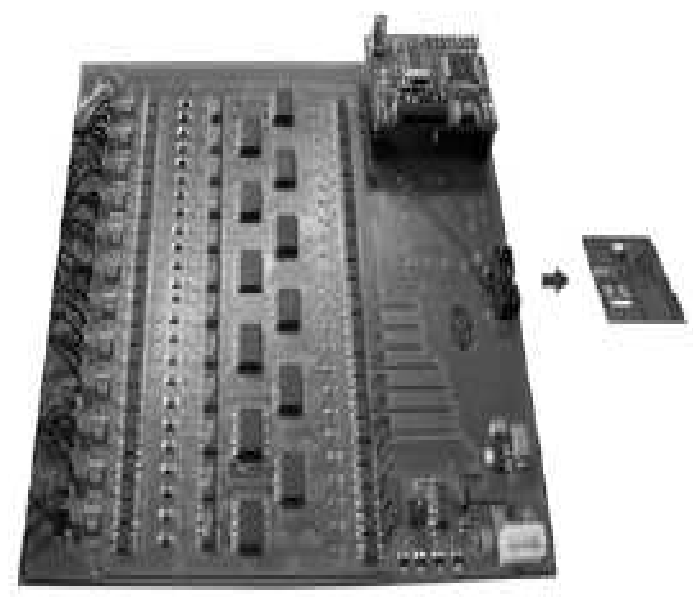

Figure 2: EEG Miniaturization.

$250 \mathrm{~Hz}$ with 16 bits implying a data rate of $4 \mathrm{kbps}$ per channel. Bluetooth [5] wireless interface is used to transmit the EEG signals to PDAs. Bluetooth supports minimum data rate of $115.2 \mathrm{kbps}$ and a maximum data rate of 921.6 kbps [6]. With the minimum data rate, all 24 channels of EEG data can be transferred using Bluetooth.

Since traditional Medium Access Protocols (MAC) cannot take the advantage of the application specific requirements of medical sensor networks, it is suggested, in [7], to use a different approach and a low power MAC layer design is presented. This MAC protocol avoids wasting energy in idle listening, collisions and power outspending in transmissions.

In a recent clinical trial, it is proved that four-channel wireless EEG quality is acceptable and feasible to perform in the hospital emergency rooms [8].

Processing of the stored EEG data on personal computers or PDAs is an integral part of EEG systems. Some commercial EEG systems enable downloading of EEG data to a central site over TCP/IP connections. It is also possible to connect the PDA to the corporate network over a wireless connection (IEEE 802.11 or GSM) and make the data and the processing software available online to the other users.

The next generation EEGs as part of the medical sensor networks can be built if the electrodes can be removed and if each channel can be implemented separately. The resulting EEG sensor can be built together with a processor and a wireless component. Fig. 2 shows the miniaturization that can be achieved if such a design approach is taken [9]. Effectively this will allow mote-based EEGs bringing many new challenges which are discussed in Section 4.

\section{MACHINE LEARNING APPROACHES}

In recent years, EEG signals have been used in BrainComputer Interface (BCI) research. BCI is oriented toward recognizing EEG patterns obtained from the human scalp and associating it with the subject's mental state. BCI systems first record EEG signals from human scalp and then extract appropriate features after removing artifacts from the recorded EEG signals. These features are then classified online using proper machine learning classifiers. The classifier results are converted into proper actions and then the subject is trained by giving feedback. An application is to automatically move a computer cursor left or right when training a handicapped person to use computers.

In our research, we have used the Bayesian [10] machine learning techniques which calculate the probabilities of variations in EEG signals by using mixture of Gaussian family of distributions. The classifier calculates the likelihood of each input signal to categorize it into a class of mental state. Here the EEG signals are the evidence and the calculated probabilities form the hypothesis for classification.

The accuracy of classification and the time taken for training are important parameters of EEG signal classification using wireless sensor networks. Accuracy is the percentage of unknown data sets that are classified to correctly belong to a mental task out of $\mathrm{N}$ tasks. In our previous study [11], we found that the Bayesian network (BNT) classifier gives the most accurate results.

\subsection{Analyzing EEG Data}

EEG signals are usually contaminated by eye movements, eye blinks, and muscular activities. Therefore, before analyzing EEG signals, we need to remove these artifacts from samples collected by electrodes. We have worked with the EEG data gathered and posted online by Z. Keirn [12]. The sample EEG data consist of five mental tasks, viz., the Baseline task, the Letter Composition task, the Multiplication task, the Counting task, and the Rotation task, for five trials from seven different subjects. Data of each task were collected from seven EEG channels c3, c4, p3, p4, o1, o2, and EOG and were taken at $250 \mathrm{~Hz}$ for 10 seconds.

We have used the Independent Component Analysis (ICA) [13] in order to remove artifacts from the EEG data collected from sensors. The ICA method is based on the assumptions that the time series recorded on the scalp are spatially stable mixtures of the activities of temporally independent cerebral and artifactual sources, that the summation of potentials arising from different parts of the brain, scalp, and body is linear at the electrodes, and that propagation delays from the sources to the electrodes are negligible.

ICA computes an 'unmixing' matrix, W, which decomposes or linearly unmixes the multi-channel scalp data into a sum of temporally independent and spatially fixed components. The rows of the output data matrix, $\mathrm{U}=\mathrm{WX}$, are time courses of activation of the ICA components. The columns of the inverse matrix, $\operatorname{inv}(\mathrm{W})$, give the relative projection strengths of the respective components at each of the scalp sensors (right). Calling the activations the matrix of unmixed component time courses,

$$
\begin{aligned}
& W=\text { weights } * \text { sphere } \\
& \text { activations }=W * \text { data }
\end{aligned}
$$

and the inverse weight matrix (i.e., the mixing matrix), $W^{-1}=i n v(W)$ then the projection of the i-th independent component onto the original data channels is given by:

$$
\text { projection }=\mathrm{W}^{-1}(:, \mathrm{i}) * \operatorname{activations}(\mathrm{i},:)
$$

In the above equations, artifact-free event-related brain signals were obtained by projecting the sum of selected nonartifactual ICA components back onto the scalp,

$$
\text { clean_data }=\mathrm{W}^{-1}(:, \mathrm{a}) * \operatorname{activations}(\mathrm{a},:)
$$

where [a] is a vector of the selected non-artifactual component numbers. 
Table 1: Classification Accuracy

\begin{tabular}{|l|l|l|l|l|l|l|l|l|}
\hline & S-1 & S-2 & S-3 & S-4 & S-5 & S-6 & S-7 & Avg \\
\hline T-1 & 5 & 4 & 5 & 5 & 3 & 5 & 5 & 91.4 \\
\hline T-2 & 3 & 5 & 5 & 5 & 4 & 5 & 5 & 91.4 \\
\hline T-3 & 5 & 5 & 4 & 4 & 5 & 4 & 5 & 91.4 \\
\hline $\mathrm{T}-4$ & 5 & 4 & 5 & 4 & 5 & 5 & 5 & 94.3 \\
\hline T-5 & 5 & 5 & 4 & 5 & 5 & 5 & 3 & 91.4 \\
\hline
\end{tabular}

After removing artifacts from the EEG data, we have extracted frequency components, i.e., features, from the clean EEG data. Two seconds long segments of the time signal which overlap one second have used to compute the power spectrum with a short time Fourier transformation [14]. This gives one feature per second and frequency band of $1 / 2 \mathrm{~Hz}$. We have used a modified MFCC [15], which has linear frequency spacing below $100 \mathrm{~Hz}$ and logarithmic spacing above $100 \mathrm{~Hz}$.

Feature values for different frequency bands might have different ranges and they might fluctuate differently. However, large fluctuations do not necessarily mean a large importance for classification [16]. Therefore, a simple normalization technique is used on training and test data sets. On training and test data sets, mean and variance are calculated for each electrode and each frequency band. The obtained values are then used for mean subtraction and variance normalization on the training and test data sets.

The goal of EEG signal classification is to recognize the mental state of interest into one of a number of mental sates. Since the classification procedure in our case is applied on extracted features, it can be also referred to as feature matching. Furthermore, if there exists some set of patterns that the individual classes of which are already known, then one has a problem in supervised pattern recognition. This is exactly our case since during the training session; we have taken EEG signals for five different mental tasks from five different subjects for the first trial. These EEG signals comprise the training set and are used to derive a classification algorithm. The EEG data for the other 5 trials, are then used to test the classification algorithm; these EEG signals are collectively referred to as the test set. The results shown in Table 1 are from the accuracy of classification done on 5 mental tasks for seven subjects and for 5 trials. The last column of the table is the average percentage of success for each task on different subjects.

In the above table, S-1 to S-7 represent seven subjects and T-1, T-2, T-3, T-4 and T-5 represent Baseline, Letter Composition, Multiplication, Counting, and Rotation tasks respectively. The performance of the system can be improved by designing a hybrid classifier. Once this optimal classifier is designed, then the resulting classifier can be trained for each subject.

\section{MEDICAL SENSOR NETWORKS WITH WIRELESS EEGS}

We assume a combined software and hardware platform for medical sensor networks such as the CodeBlue [17]. The CodeBlue provides several features that are useful for EEGs: location tracking, publish/subscribe routing layer and query interface. RF-based location tracking in CodeBlue allows mobility to the patients with EEG sensors in their scalp.
Publish/subscribe routing layer is based on the adaptive demand-driven multicast routing (ADMR). With ADMR, the requests from several medical personnel for a given patient's EEG data can be multicast efficiently over the adhoc network created by several mote-based sensors that the patients in the hospital will carry. The CodeBlue query interface $(\mathrm{CBQ})$ is how the end-user devices in the medical sensor network express their data requirements from the sensor nodes such as EEG sensors.

Wireless link in mote-based EEGs can be implemented using Bluetooth, i.e. IEEE 802.15.1. Mote sensors designed with Bluetooth radio running TinyOS have been reported in the literature [18]. Since, the CodeBlue uses Telos B motes we envisage implementing EEG sensors using Tmote [19] motes with IEEE 802.15 .4 radio and 12 bit A/D conversion hardware. EEG data will be digitized and stored in Tmote's flash memory. EEG data can then be transmitted to a nearby base station (a Stargate with a Tmote) using a reliable communication protocol which is part of the CodeBlue. In our system, we have used Stargates as the base stations, Stargates have the processing power and memory capabilities of a typical PDA.

For several EEG sensors in the patient's scalp, the data from each sensor node need to be synchronized at the base station. For this purpose the data are time-stamped. There is a similar synchronization problem when motion analysis sensors are used on human body. A patient will wear several Tmote motes outfitted with motion analysis sensors and the data from these sensors arriving at the base station need to be correlated [20].

The use of Tmote motes together with Stargates and more powerful base station for processing EEG data leads to a three level hybrid system design. Our hybrid system architecture is shown in Fig. 3. A similar but two-level approach was used in an environmental sensor network application [21]. Our system is unique in that EEG data is much more complicated than processing audio signals coming from the environment.

In the our system, we have uploaded EEG data into seven Tmote motes; we have used one mote to store data from one channel for five mental tasks and for five trials. The $\mathrm{NesC}$ [22] application on motes, will transmit EEG samples based one our query, e.g., read $\mathrm{N}$ samples for task $\mathrm{T}$ for subject $\mathrm{S}$.

The user interface module is used to put input queries to the system and is a Java application runs on PDAs or PCs and to display query results on the screen. The query processor parses queries and generates appropriate commands for the sensors or the Machine learning system. The routing module forwards the query to the appropriate sensors and query results back to the base station. The sensor interface interacts with the sensor nodes, i.e., gets sensor readings and delivers data to the routing module. The Machine Learning subsystem performs a number of things including artifact removal, Fourier transformation, feature extraction, and task classification.

\subsection{Query Processing}

We have designed a simple query processor for our wireless EEG system which is adapted from the CodeBlue, queries take the form

$$
\langle S, C, p\rangle
$$

where $\mathrm{S}$ is the set of node IDs that should report data for 


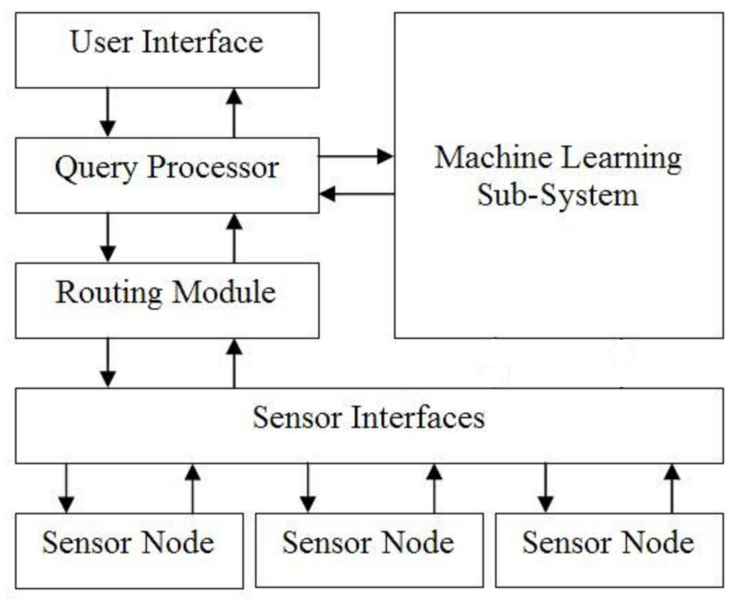

Figure 3: TinyOS EEG Implementation.

this query, $\mathrm{C}$ is the count of the total number of samples and can be left unspecified to obtain the samples continuously and $\mathrm{p}$ is the filter predicate.

By incorporating classification based on Bayesian machine learning algorithm into the system, we have added additional queries based on mental tasks, such as counting or rotation task. In our system, the query predicates can specify the mental task being performed by the subject, for example

$$
\langle S,, p=T-1\rangle
$$

. For this type of query, the query processor sends a query to the motes for subject $\mathrm{S}$ and the data received from motes are then forwarded to the machine learning subsystem for recognition. The machine learning system removes artifacts from received EEG data, extracts EEG features from the received data, runs the Bayesian classifier on the EEG features. The result of the machine learning system, i.e., the recognized task, is then sent to the user interface module to display. Note that for machine learning classification, we have trained a Bayesian classifier using sample EEG data before sensor deployment. The efficient implementation of these algorithms in real time is the major challenge in obtaining the results on timely fashion.

\subsection{Multihop Routing}

A simple tree based multihop routing algorithm has been implemented in our system for forwarding queries and results. The base station is the root of the tree and all communication is gone through the root. The root periodically broadcasts beacon messages with monotonically increasing sequence number. The first hop nodes that receive beacon message set base station as their parents and forward the beacon message to the next hop nodes. The children notify their parents to make the tree loop free. Every node always selects the least cost root from the beacon message. The base station broadcasts queries and the source node always unicasts packets to its parent toward the base station.

\section{CONCLUSIONS}

We have presented the state-of-the-art of wireless EEGs which transmit the brain waves to a personal computer or
PDA nearby for further processing. We have shown that wireless EEGs can be incorporated in the medical sensor networks. The hardware needed is TinyOS based sensor nodes equipped with EEG sensors. We have also presented a three-level hybrid medical sensor network design in which Tmote Sky mote-based EEG sensors are used at the lowest level, Stargates are used at the second level and more powerful PCs are used at the highest level. The system also contains other types of general-purpose PDAs that the users, medical personnel carry.

We have used the Bayesian network as the EEG signal classifier which gives the most accurate result as shown in our previous study. We have also designed a query processing subsystem and a multihop routing module for the hybrid medical sensor network. The queries initiated by generalpurpose PDAs are processed by the base stations that have EEG data from the EEG sensors that the patients carry.

The future challenges include designing the mote-based EEG sensor nodes to handle brain monitoring. Implementation of the extensions we developed to CodeBlue for motebased EEGs is needed to establish a prototype platform with which more experimentation can be done. Many new research directions lie ahead especially in machine intelligence algorithm development as the data from the brain sensors is coming online from the most complicated human organ including reducing the complexity of the classifiers. Networking issues such as fairness, latency, packet jitter and the effects of patient mobility to online monitoring need to be investigated.

\section{REFERENCES}

[1] K. Romer and F. Mattern, "The design space of wireless sensor networks," IEEE Wireless Communications Magazine, vol. 11, no. 6, pp. 54-61, December 2004.

[2] J. Hill, R. Szewczyk, A. Woo, S. Hollar, D. Culler, and K. Pister, "System architecture directions for networked sensors," in Proc. of 9th Intl. Conf. on Architectural Support for Programming Languages and Operating Systems, Boston, MA, USA, Nov 2000, pp. 93-104.

[3] IEEE, IEEE 802.15.4 - Wireless Medium Access Control and Physical Layer Specifications for $L R$-WPANs, October 2003.

[4] Honal M. and Schultz T., "Identifying user state using electroencephalographic data," in In Proceedings of the ICMI 2005 Workshop, Trento, Italy, October 2005.

[5] J. C. Haartsen and Ericsson Radio System B.V., "The bluetooth radio system," IEEE Personal Communications, February 2000.

[6] N. Baisa, "Designing wireless interfaces for patient monitoring equipment," RFDesign, pp. 1-5, April 2005. [Online]. Available: http://rfdesign.com/

[7] I. E. Lamprinos, A. Prentza, E. Sakka, and D. Koutsouris, "A low power medium access control protocol for wireless medical sensor networks," in Proceedings of the 26th Annual International Conference of the IEEE EMBS, San Francisco, USA, Sep 2005.

[8] Bastani, Aveh, Medado, Patrick, Qadir, Rizwan, Manthena, and Prasanth, "The feasibility of acquiring and transmitting real-time wireless eegs in the 
emergency department," Acad Emerg Med, vol. 12, no. 5_suppl_1, pp. 172-a-, 2005.

[9] IMEC Inc., "Ambulatory EEG," IMEC Human ++ Program, pp. 1-2, 2005. [Online]. Available: http://www.imec.be

[10] Friedman N. and Goldszmidt M., "Building classifiers using bayesian networks," in In Proceedings of the Thirteenth National Conference on Artificial Intelligence, Menlo Park, CA, USA, 1996.

[11] S. Rezaei, K. Tavakolian, and K. Naziripour, "Comparison of five different classifiers for classification of mental tasks," in Proc. of the 2005 IEEE Engineering in Medicine and Biology, Shanghai, China, Sep 2005, 4p.

[12] Z. Keirn, "Classification of EEG signals for brain-machine interfaces," Colorado State University, 2003. [Online]. Available: http://www.cs.colostate.edu/eeg/

[13] S. Makeig, M. Masterfield, and Tzyy-Ping Jung, "ICA Toolbox Tutorial," University of California-San Diego, 2001. [Online]. Available: http://www.sccn.ucsd.edu/ ${ }^{\text {scott/tutorial/ }}$

[14] E. Brigham, Fast Fourier Transform and Its Applications. Prentice Hall Inc., 1988, no. ISBN 0-13-307505-2.

[15] Young S., Evermann G., Kershaw D., Moore G., Odell J., Ollason D., Valtchev V., and Woodland P., "The HTK book," November 2001.

[16] Wallerius J., Trejo L. J., Matthews R., Rosipal R. and Caldwell J. A., "Robust feature extraction and classification of eeg spectra for real-time classification of cognitive state," in Proceedings of 11th

International Conference on Human Computer Interaction, Las Vegas, NV, USA, July 2005.

[17] K. Lorincz, D. Malan, V. Shnayder, and M. Welsh, "Sensor networks for emergency response: Challanges and opportunities," IEEE Pervasive Computing Magazine, vol. 3, no. 4, pp. 16-23, October-December 2004.

[18] J. Beutel, M. Dyer, L. Meier, and L. Thiele, "Scalable topology control for deployment-support networks," in Proc. of IEEE IPSN 2005, Los Angeles, USA, Apr. 2005, pp. 359-363.

[19] Moteiv Inc., Tmote Sky.

[20] V. Shnayder, Bor-rong Chen, K. Lorincz, Thaddeus R.F., Fulford-Jones, and M. Welsh, "Sensor networks for medical care," Technical Report, Harvard University, April 2005, 14p.

[21] W. Hu, V. N. Tran, N. Bulusu, C. T. Chou, S. Jha, and A. Taylor, "The design and evaluation of a hybrid sensor network for cane-toad monitoring," in Proc. of IEEE IPSN 2005, Los Angeles, USA, Apr. 2005.

[22] D. Gay, P. Levis, R. von Behren, E. Brewer, M. Welsh, and D. Culler, "The nesc language: A holistic approach to network embedded systems," in In ACM SIGPLAN Conference on Programming Language Design and Implementation, San Diego, CA, USA, June 2003. 